\title{
Schlechte Prognose auch bei hustenden Rauchern mit normaler Lungenfunktion
}

\begin{abstract}
Hintergrund und Fragestellung: Eine chronisch obstruktive Lungenerkrankung (COPD) wird gemäß der Global Initiative for Chronic Obstruction Lung Disease (GOLD-)Stadien je nach Ergebnis der Sekundenkapaziätsmessung in der Spirometrie eingeteilt. Viele Raucher haben noch eine normale Lungenfunktion, jedoch dennoch Symptome wie Husten, Auswurf und Kurzatmigkeit, die auf eine COPD hinweisend. Diese Studie stellte die Hypothese auf, dass Raucher mit einer respiratorischen Symptomatik und normalen Lungenfunktion einen ähnlichen klinischen Verlauf und Befunde wie bei COPD haben.
\end{abstract}

Patienten und Methoden: Zwischen 2010 und 2015 wurden in der multizentrischen Beobachtungsstudie 2736 Patienten zwischen 40 und 80 Jahren aufgenommen, die entweder nie geraucht haben oder eine positive Raucheranamnese mit 20 Schachteljahren hatten. Das Durch-

\section{Originalie}

Woodruff PG, Barr RG, Bleecker E et al. Clinical Significance of Symptoms in Smokers with Preserved Pulmonary Function. N Engl J Med. 2016;374(19):1811-21 schnittsalter der Teilnehmer lag bei 56,4 Jahren in der Nichtrauchergruppe, 61,9 bzw. 59,3 Jahren in der Rauchergruppe mit normaler Lungenfunktion und 67,9 bzw. 64,9 Jahren in der Rauchergruppe mit COPD. Sie wurden je nach Symptomatik mittels eines evaluierten Tests, der die Atemwegssymptomatik erfasste (CAT= COPD Assessment Test), und dem Ergebnis der Spirometrie stratifiziert. Alle 3 Monate wurde mit Erhebung dieser Parameter nachuntersucht sowie dem 6-Minuten-Gehtest und einer CT-Untersuchung der Lunge.

Ergebnisse: Die Hälfte der Raucher mit normaler Lungenfunktion hatten Symptome im CAT. Im Nachbeobachtungszeitraum von 820 Tagen (IR 494-1106) hatten mehr Raucher mit Symptomen eine Exazerbation als Raucher ohne Symptome oder Nichtraucher $(0,27 \pm 0,67$ vs. $0,08 \pm 0,31$ und $0,03 \pm 0,21$ Ereignisse, $\mathrm{p}<0,001)$. Die Gehstrecke war bei Rauchern mit Symptomen kürzer als in den anderen beiden Gruppen, im CT waren signifikant mehr Hinweise auf die Entwicklung eines Emphysems in der Gruppe der Raucher mit Symptomatik versus der Raucher ohne Symptomatik.

Schlussfolgerung: Diese longitudinale Studie zeigte einen hohen Anteil an Rauchern mit respiratorischen Symptomen und normaler Lungenfunktion an. Diese hatten ein höheres Risiko für Exazerbationen, eine kürzere Gehleistung, Hinweise für eine Verdickung der Atemwege und der sogenannten stillen Atemwegserkrankung (nicht durch Veränderung der Sekundenkapazitätsmessung feststellbar).

\section{- Kommentar von Prim. Dr. Peter Dovjak und Prof. Dr. med. Hans J. Heppner}

\section{Symptom-Fragebogen sinnvoll!}

Die Spirometrie allein kann das Risiko für die Entwicklung einer Exazerbation der Atemwegserkrankung, den erhöhten Bedarf an antibiotischer Therapie und Kortison nicht erfassen [1]. Zusätzlich ist in der Überwachung von Rauchern die Erfassung der Symptomatik wichtig, z.B. mittels eines evaluierten Fragebogens, der 2009 als kurzer, einfacher Test in die klinische Praxis eingeführt wurde [2]. Die Konsequenzen für Raucher mit normaler Lungenfunktion und vorhandener Symptomatik gleichen jenen, die an milder bis mittlerer Ausprägung einer COPD erkrankt sind. Das stellt einen wesentlichen Paradigmenwechsel für die Therapieplanung dar.

\section{Literatur}

1. Fabbri LM. Smoking, Not COPD, as the Disease. N Engl J Med. 2016;374(19):1885-6. Die giftigen Rauchgase schädigen die Lunge, auch wenn die Spirometrie noch unauffällig ist.

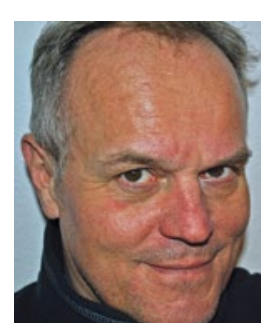

Prim. Dr. Peter Dovjak

Leiter der Akutgeriatrie

Salzkammergutklinikum Gmunden

Miller von Aichholzstraße 49

A-4810 Gmunden

peter.dovjak@gespag.at

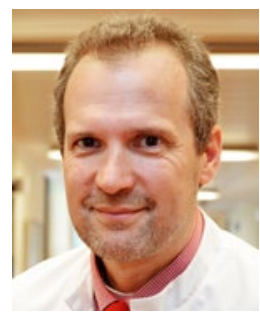

Univ.- Prof. Dr. med. Hans Jürgen Heppner

Chefarzt Geriatrische Klinik und Tagesklinik Lehrstuhl für Geriatrie

Universität Witten/Herdecke

Dr.-Moeller-Straße 15, 58332 Schwelm

hans.heppner@uni-wh.de 